\title{
Comparison between single antiplatelet therapy and combination of anti- platelet and anticoagulation therapy for secondary prevention in ischemic stroke patients with antiphospholipid syndrome
}

\author{
Hirohisa Okuma ${ }^{1 凶}$, Yasuhisa Kitagawa ${ }^{2}$, Takashi Yasuda ${ }^{2}$, Kentaro Tokuoka ${ }^{2}$, Shigeharu Takagi ${ }^{3}$ \\ 1. Department of Neurology, Tokai University Tokyo Hospital; \\ 2. Department of Neurology, Tokai University Hachioji Hospital; \\ 3. Department of Neurology, Tokai University School of Medicine.
}

Correspondence to: Hirohisa Okuma, Department of Neurology, Tokai University Tokyo Hospital, 1-2-5 Shibuya-ku, Yoyogi, Tokyo 151-0053, Japan. Tel: +81-3-3370-2321, Fax: +81-3-3370-2321, E-mail: ookuma@tok.u-tokai.ac.jp

Received: 2009.10.02; Accepted: 2009.11.30; Published: 2009.12.05

\begin{abstract}
Satisfactory results have not yet been obtained in therapy for secondary prevention in ischemic stroke patients with antiphospholipid syndrome (APS). We therefore compared single antiplatelet therapy and a combination of antiplatelet and anticoagulation therapy for secondary prevention in ischemic stroke patients with APS.

The subjects were 20 ischemic stroke patients with antiphospholipid antibody, 13 with primary antiphospholipid syndrome and 7 with SLE-related antiphospholipid syndrome. Diagnosis of APS was based on the 2006 Sydney criteria. Eligible patients were randomly assigned to either single antiplatelet therapy (aspirin $100 \mathrm{mg}$ ) or a combination of antiplatelet and anticoagulation therapy (target INR: 2.0-3.0; mean 2.4 \pm 0.3 ) for the secondary prevention of stroke according to a double-blind protocol. There was no significant difference between the two groups in age, gender, NIH Stroke Scale on admission, mRS at discharge, or rate of hypertension, diabetes mellitus, hyperlipidemia, or cardiac disease. We obtained Kaplan-Meier survival curves for each treatment. The primary outcome was the occurrence of stroke. The mean follow-up time was $3.9 \pm 2.0$ years. The cumulative incidence of stroke in patients with single antiplatelet treatment was statistically significantly higher than that in patients receiving the combination of antiplatelet and anticoagulation therapy (log-rank test, $\mathrm{p}$-value $=0.026$ ). The incidence of hemorrhagic complications was similar in the two groups. The recent APASS study did not show any difference in effectiveness for secondary prevention between single antiplatelet (aspirin) and single anticoagulant (warfarin) therapy. Our results indicate that combination therapy may be more effective in APS-related ischemic stroke.
\end{abstract}

Key words: antiphospholipid syndrome, APS-related ischemic stroke, single antiplatelet therapy, combination therapy, Kaplan-Meier survival curves.

\section{Introduction}

Antiphospholipid syndrome (APS) [1] is a common autoimmune prothrombotic condition characterized by arterial and venous thrombosis and pregnancy morbidity, associated with persistently positive anticardiolipin antibodies (aCL) and/or lupus anticoagulant (LA) [2]. Concerning therapy, satisfactory results have not yet been obtained in therapy for secondary prevention in ischemic stroke patients 
with APS. We therefore compared single antiplatelet therapy and a combination of antiplatelet and anticoagulation therapy for secondary prevention in ischemic stroke patients with APS. According to the guidelines of the American Heart Association (APASS) [3] for prevention of stroke in patients with ischemic stroke or transient ischemic attack and with antiphospholipid antibodies (aPL), antiplatelet therapy is reasonable for cases of cryptogenic ischemic stroke or TIA with positive aPL. On the other hand, oral anticoagulation with a target INR of 2 to 3 [4] is reasonable for patients with ischemic stroke or TIA who meet the criteria for APS with venous and arterial occlusive disease in multiple organs, miscarriages, and livedo reticularis.

\section{Materials and Methods}

We focused on the secondary prevention of stroke with APS, and compared single antiplatelet therapy and a combination of antiplatelet and anticoagulation therapy in ischemic stroke patients with APS. The subjects were 20 ischemic stroke patients with antiphospholipid antibody (10 males and 10 females, mean age 48 years), who were hospitalized between October 2002 and November 2004.

They consisted of 13 with primary antiphospholipid syndrome and 7 with SLE-related antiphospholipid syndrome. Diagnosis of APS was based on the 2006 Sydney criteria [5]. Only patients with positive IgG beta 2 glycoprotein I (beta 2-GPI)-dependent anticardiolipin antibody and/or lupus anticoagulant, present on two or more occasions, six weeks or more apart, were selected. Eligible patients were randomly assigned to either single antiplatelet therapy (aspirin $100 \mathrm{mg}$ ) [6] or a combination of antiplatelet and anticoagulation therapy (target INR: 2.0-3.0; mean 2.4 \pm 0.3 ) for the secondary preven- tion of stroke, according to a double-blind protocol [3, 7]. The purpose of the present study was to examine the effects of these regimens on recurrence of stroke. So, the primary endpoint was occurrence of stroke.

This study was approved by the ethics committee of Tokai University, and prior informed consent was obtained from all patients who were eligible to participate. Randomization was performed using a randomly generated score.

\section{Results}

Table 1 shows the background of the two groups. There was no significant difference between the two groups in age, gender, NIH Stroke Scale on admission, modified Rankin scale (mRS) at discharge, or rates of hypertension, diabetes mellitus, hyperlipidemia, and cardiac disease. Transthoracic cardiac echo findings were available for 15 patients. The echocardiograms detected three mitral valve abnormalities, but these were not thought to be potential embolic sources. Two of these patients were randomized to the combination therapy group, and the other to the single modality group.

Kaplan-Meier survival curves are shown in Figure 1. The mean follow-up time was $3.9 \pm 2.0$ years. The cumulative incidence of stroke in patients with single antiplatelet treatment was higher than that in patients receiving the combination of antiplatelet and anticoagulation therapy (log-rank test, $p$-value $=0.026)$. This difference is statistically significant. However, the patient who had recurrent thrombotic infarction in the combination of antiplatelet and anticoagulation therapy group showed an INR before the recurrence of 2.0 , so the possibility of inadequate treatment can not be ruled out.

Table I. Baseline characteristics of patients.

$$
\begin{gathered}
\text { Group } 1 \\
\text { Single antipletelet therapy } \\
(\mathrm{n}=11)
\end{gathered}
$$

Group 2

Combination of therapy antiplatelet and anticoagulant therapy

$(n=9)$
Mean age (ys)

Female (\%)

NIHSS (at admission)

mRS (at discharge )

Hypertension (\%)

Diabetes mellitus (\%)

Hyperlipidemia (\%)

Atrial fibrillation(\%)

SLE/APS (\%)
47

50

4.8

1.9

63.6

18.2

18.2

9.1

36.4

\section{9}

50

5.2

2.1

55.6

22.2

22.2

11.1

33.3 p-value

ns

ns

ns

ns

ns

ns

ns

ns

ns 


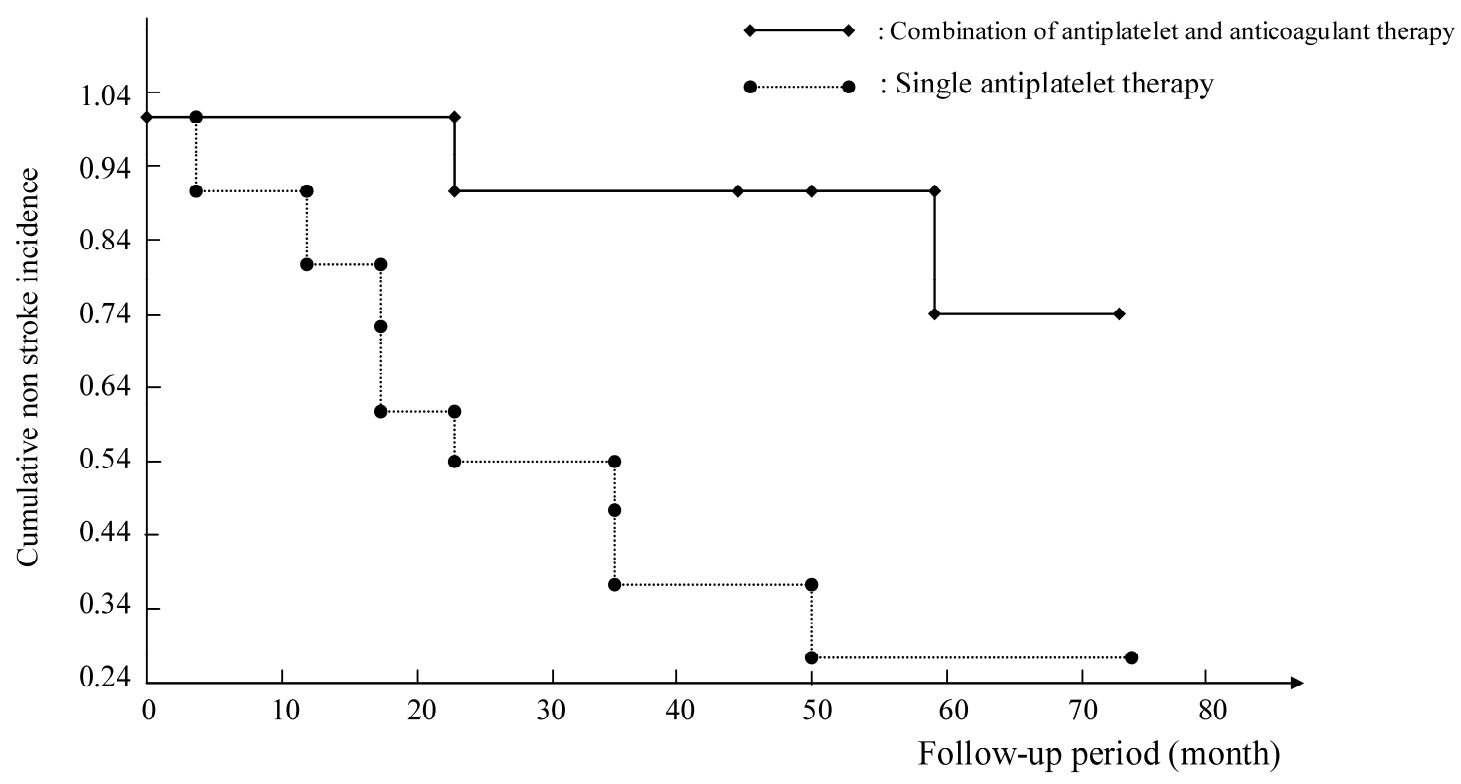

Figure I. Comparison between single antiplatelet therapy and combination of antiplatelet and anticoagulation therapy for secondary prevention in ischemic stroke patients with antiphospholipid syndrome.

Table 2. Hemorrhagic complications.

\begin{tabular}{lcc}
\hline Group 1: Single antiplatelet & $\begin{array}{r}\text { Group 2: Combination of } \\
\text { antiplatelet and } \\
\text { anticoagulant } \\
(\mathrm{N}=9)\end{array}$ \\
\hline Cerebral hemorrhage & 1 & 0 \\
GI bleeding & 0 & 0 \\
Subcutaneous hemorrhage & 0 & 1 \\
\hline
\end{tabular}

Next, we examined hemorrhagic complications in both groups. One minor cerebral hemorrhage was noted in the single antiplatelet therapy group, and one subcutaneous hemorrhage was found in the combination therapy group. As for the patient in the single antiplatelet therapy group who developed cerebral hemorrhage, magnetic resonance angiography of the head showed no apparent aneurysm that might have resulted in hemorrhage. The patient was treated for hypertension, but had no other concurrent conditions. The blood pressure, at least on outpatient visits, had been stable. We did not encounter gastrointestinal bleeding. The incidence of hemorrhagic complications was similar in the two groups (Table 2).

\section{Discussion}

There is still debate as to which therapy is the most effective for secondary prevention of stroke with APS $[4,8,9,10]$ and concerning the relationship be- tween APS and stroke. It is generally accepted that aPL is an independent risk factor for initial ischemic stroke in young adults [11, 12].

Treatment to prevent recurrent stroke and other thrombotic events in APS patients has been reviewed [13]. Two groups have retrospective data to suggest that high-intensity warfarin treatment is associated with a better outcome in selected cohorts with various types of thrombotic events [6]. Khamashta [14] reported that high-intensity warfarin (INR over 3.0) with or without low-dose aspirin (75 mg/day) was significantly more effective than low-intensity warfarin (INR under 3.0) with or without low-dose aspirin, or treatment with aspirin alone, in preventing further thrombotic events. Crowther [7] recently reported the results of the first randomized, double-blind, controlled trial of two different intensities of warfarin treatment on the prevention of recurrent thrombotic events in patients with APS. The high-intensity warfarin treatment was no more effective than moderate-intensity treatment in preventing recurrent thrombotic events.

The APASS study [3] was the first prospective study of the role of aPL in recurrent ischemic stroke, in collaboration with the WAPS group [8]. This study did not show any difference in effectiveness for secondary prevention between single antiplatelet (aspirin) and single anticoagulant (warfarin) therapy. Derksen [15] examined the effect of low-dose aspirin after first ischemic stroke associated with aPL. During about 9 years of follow-up, 2 of 9 patients had a re- 
current stroke. Recurrent stroke rate per 100 patient-years on aspirin was only 3.5. But, we think single antiplatelet therapy may be less effective for the secondary prevention of stroke than the combination of antiplatelet and anticoagulant therapy. We have examined endothelial function in patients with APS. Although protein $C$ is activated on endothelial cells [16], we found that serum obtained from patients with positive $\operatorname{IgG}$ cardiolipin antibodies interfered with protein $C$ activation [2]. Protein $C$ activation is disturbed in patients with APS [17]. Since protein C is closely associated with factor VIII and factor V, this result suggests that the coagulation system is impaired in patients with APS, and so anticoagulant could be effective. Aspirin influences endothelial function, and although the effect may be dose-dependent, the dose of $100 \mathrm{mg}$ may be sufficient to improve endothelial function.

There are some important limitations to be considered. First, diagnosis of APS in WAPS [8] was not based on the 2006 Sydney criteria [5]. As only a single measurement of anticardiolipin antibody and LA was obtained, cases with IgG beta-2 GPI non-dependent cardiolipin antibody were included. Second, the average age of patients (63 years) was significantly older than that in typical APS studies (34 years). Third, the target INR in WAPS [8] was for cardiogenic stroke caused by non-valvular Af. The dosage of aspirin in WAPS [8] was $325 \mathrm{mg}$. Currently, the recommended dosage of aspirin is only $75-150 \mathrm{mg}$. Treatment recommendations in this study were based on secondary prevention of ischemic stroke in patients without associated aPL. The patients in our study were selected according to the Sydney criteria [5] of APS and the average age was consistent with that in typical APS patients.

There have not been any previous studies dealing with the combination of antiplatelet and anticoagulant therapy for ischemic stroke patients with APS based on the strict Sydney criteria [5]. One reason may be that patients with stroke complicated with APS are rather rare compared with patients with uncomplicated stroke, and this is also the reason why the number of patients in this study was quite small. Nevertheless our results seem promising, and a larger study with more patients would be warranted.

\section{Conclusion}

Our results indicate that a combination of antiplatelet and anticoagulation therapy may be more effective than single antiplatelet therapy for secondary prevention in ischemic stroke patients with APS.

\section{Conflict of Interest}

The authors have declared that no conflict of interest exists.

\section{References}

1. Harris EN, Gharavi AE, Boey ML, et al. Anticardiolipin antibodies: detection by radioimmunoassay and association with thrombosis in systemic lupus erythematosus. Lancet. 1983; 2: 1211-4.

2. Kitagawa Y. Antiphospholipid antibodies syndrome and ischemic stroke. Annual Review. 2004; 1: 139-51.

3. Sacco RL, Adams R, Albers G, et al. Guideline for prevention of stroke in patients with ischemic stroke or transient ischemic attack. A statement for healthcare professionals from the American Heart Association/America Stroke Association Council in. Stroke. 2006; 37: 577-617.

4. Greaves M, Cohen H, MacHi SJ, et al. Guidelines on the investigation and management of the antiphospholipid syndrome. Br J Haematol. 2000; 109: 704-15.

5. Miyakis S, Lockshin MD, Atsumi T, et al. International consensus statement on an update of the classification criteria for definite antiphospholipid syndrome (APS). J Thromb Haemost. 2006; 4: 295-306.

6. Jacobs BS, Levine SR. Antiphospholipid antibody syndrome. Cure Treat Options Neurol. 2000; 2: 449-58.

7. Crowther MA, Ginsberg JS, Julian J, et al. A comparison of two intensities of warfarin for the prevention of recurrent thrombosis in patients with the antiphospholipid antibody syndrome. N Eng J Med. 2003; 349: 1133-8.

8. Finazzi G, Marchioli R, Brancaccio V, et al. A randomized clinical trial of high-intensity warfarin vs. conventional antithrombotic therapy for the prevention of recurrent thrombosis in patients with the antiphospholipid syndrome (WAPS). J Thromb Haemost. 2005; 3: 848-53.

9. Wittkowsky AK, Downing J, Blackburn J, et al. Warfarin-related outcome in patients with antiphospholipid antibody syndrome managed in an anticoagulation clinic. Throb Haemost. 2006; 96: 137-41.

10. Erkan D, Lockshin MD. New approaches for managing antiphospholipid syndrome. Nat Clin Pract Rheumathol. 2009; 5: 160-70.

11. Okuma H, Kitagawa Y, Takagi S, et al. Prevalence rates of antiphospholipid antibodies in ischemic stroke patients. Intern. Med. 2006; 45: 1017-8.

12. Diz-Kucukkaya R, Hancer VS, Artin-Esen B, et al. The prevalence and clinical significance of inherited thrombophilic risk factors in patients with antiphospholipid syndrome. J Thromb Thrombolysis. 2009; 6: 356-9.

13. Brey RL. Management of the neurological manifestations of APS - what do the trials tell us? Thrombosis Research. 2004; 114: 489-99.

14. Khamashta MA, Cuadrado MJ, Mujic F, et al. The management of thrombosis in the antiphospholipid-antibody syndrome. $\mathrm{N}$ Eng J Med. 1995; 332: 993-7.

15. Derksen RH, Groot PG, Kappelle LJ. Low dose aspirin after ischemic stroke associated with antiphospholipid syndrome. Neurology. 2003; 61: 111-4.

16. Wahl D, Membre A, Perret-Guillaume C, et al. Mechanisms of antiphospholipid-induced thrombosis: effects on the protein $\mathrm{C}$ system. Curr Rheumatol Rep. 2009; 11: 77-81.

17. Li $\mathrm{TH}, \mathrm{Chen} \mathrm{TH}$, Lin HS, et al. Uncoupling of protein $\mathrm{C}$ and antithrombin $\amalg$ activitiy in cerebral ischemia patients with cutis marmorata. Acta Neurol Taiwan. 2008; 17: 233-8. 